\title{
Seeing the forest for the trees: Putting multilayer networks to work for community ecology
}

\author{
Matthew C. Hutchinson ${ }^{1}$ (D) | Bernat Bramon Mora ${ }^{2}$ | Shai Pilosof ${ }^{3}$ | Allison K. Barner ${ }^{4}$ \\ Sonia Kéfi ${ }^{5}$ | Elisa Thébault ${ }^{6}$ | Pedro Jordano ${ }^{7}$ (D) | Daniel B. Stouffer ${ }^{2}$ (D)
}

${ }^{1}$ Department of Ecology and Evolutionary Biology, Princeton University, Princeton, New Jersey; ${ }^{2}$ Centre for Integrative Ecology, School of Biological Sciences, University of Canterbury, Christchurch, New Zealand; ${ }^{3}$ Department of Ecology \& Evolution, University of Chicago, Chicago, Illinois; ${ }^{4}$ Department of Environmental Science, Policy, and Management, University of California Berkeley, Berkeley, California; ${ }^{5}$ ISEM, CNRS, Univ. Montpellier, IRD, EPHE, Montpellier, France; ${ }^{6} \mathrm{CNRS}$, Sorbonne Université, Institute of Ecology and Environmental Sciences of Paris, Paris, France and ${ }^{7}$ Department of Integrative Ecology, Estación Biológica de Doñana (EBD-CSIC), Seville, Spain

\section{Correspondence}

Matthew C. Hutchinson

Email: mcch@princeton.edu

\section{Funding information}

Agence Nationale de la Recherche, Grant/ Award Number: 14-CE02-0012; James S. McDonnell Foundation; Inspire Foundation; Royal Society of New Zealand; Ministerio de Economía y Competitividad, Grant/Award Number: CGL2017-82847-P

Handling Editor: Oscar Godoy

\section{Abstract}

1. A framework for the description and analysis of multilayer networks is established in statistical physics, and calls are increasing for their adoption by community ecologists. Multilayer networks in community ecology will allow space, time and multiple interaction types to be incorporated into species interaction networks.

2. While the multilayer network framework is applicable to ecological questions, it is one thing to be able to describe ecological communities as multilayer networks and another for multilayer networks to actually prove useful for answering ecological questions. Importantly, documenting multilayer network structure requires substantially greater empirical investment than standard ecological networks. In response, we argue that this additional effort is worthwhile and describe a series of research lines where we expect multilayer networks will generate the greatest impact.

3. Inter-layer edges are the key component that differentiate multilayer networks from standard ecological networks. Inter-layer edges join different networkstermed layers-together and represent ecological processes central to the species interactions studied (e.g., inter-layer edges representing movement for networks separated in space). Inter-layer edges may take a variety of forms, be species- or network-specific, and be measured with a large suite of empirical techniques. Additionally, the sheer size of ecological multilayer networks also requires some changes to empirical data collection around interaction quantification, collaborative efforts and collation in public databases.

4. Network ecology has already touched on a wide swath of ecology and evolutionary biology. Because network stability and patterns of species linkage are the most developed areas of network ecology, they are a natural starting place for multilayer investigations. However, multilayer networks will also provide novel insights to niche partitioning, the connection between traits and species' interactions, and even the geographic mosaic of co-evolution. 
5. Synthesis. Multilayer networks provide a formal way to bring together the study of species interaction networks and the processes that influence them. However, describing inter-layer edges and the increasing amounts of data required represent challenges. The pay-off for added investment will be ecological networks that describe the composition and capture the dynamics of ecological communities more completely and, consequently, have greater power for understanding the patterns and processes that underpin diversity in ecological communities.

KEYWORDS

ecological community, ecological network, food webs, interaction turnover, multilayer, multiplex, species interactions

\section{1 | INTRODUCTION}

It has been stated that "no theory of the cell, of social media or of the Internet can ignore the profound network effects that their interconnectedness cause" (Barabási, 2011). Many ecologists would argue that one paradigmatic example is noticeably missing from this list: the ecological community. After all, interactions are a defining characteristic of every species' "milieu" since no individual organism exists without participating in some sort of ecologically relevant interspecific interaction during its lifetime. Interactions between species underpin community ecology to the extent that they have even been claimed to represent "the architecture of biodiversity" (Bascompte \& Jordano, 2013).

Indeed, scholarly accounts of the myriad connections between species date back at least to al-Jāhiz in the 9th century or Aristotle in the 4th century BCE (Egerton, 2012). More pertinently, network ecology has seen massive growth in the past 40 years. Fundamental food web theory has been built on with empirical observation and experimentation (Pascual \& Dunne, 2006), a vibrant literature on bipartite interaction networks has emerged around mutualisms (Bascompte $\&$ Jordano, 2013), and models to predict species linkage have gained realism and accuracy (Eklöf et al., 2013). While this literature has generated steady progress in our understanding of ecological communities, it has relied on data representing single points in space and time, or a narrow picture of a guild's biotic interactions. Collecting sets of networks has been one way to overcome this issue (Ponisio, Gaiarsa, \& Kremen, 2017) but does not account for the dependency between networks. A new tool-multilayer networks-has recently entered the fray with the promise of addressing these issues, but key questions around their use remain unresolved.

The theoretical framework for multilayer networks (Boccaletti et al., 2014; Kivelä et al., 2014) and their implementation in ecology (Pilosof, Porter, Pascual, \& Kéfi, 2017) already exist. Indeed, theoretical contributions are already helping to bridge multilayer networks and ecological theory (García-Callejas, Molowny-Horas, \& Araújo, 2018; Godoy, Bartomeus, Rohr, \& Saavedra, 2018). Briefly, multilayer networks provide a way to encode different entities and/or interactions in a single network by distinguishing between types of network edges (Boccaletti et al., 2014; Kivelä et al., 2014). Ecological multilayer networks (hereafter, EMNs) will typically represent species interactions through time and space, or across types of interactions (Pilosof et al., 2017). For example, spatial and temporal EMNs can consist of several networks of a focal species interaction type (e.g., plant-pollinator or host-parasite interactions, with each network referred to as a layer composed of intra-layer edges) linked to each other via inter-layer edges that describe an additional ecological process, such as gene flow between habitats or change in abundance through time. EMNs composed of different types of species interactions (e.g., pollination, seed-dispersal, folivory) can also be represented as multilayer networks (hereafter, multiplex EMNs). Inter-layer edges in multiplex EMNs may represent the effect of one interaction type on the other (but see GraciaLázaro, Hernández, Borge-Holthoefer, \& Moreno, 2018). Using both intra- and inter-layer edges, EMNs enable multiple descriptors of ecological communities to be linked together into a single network. That network will capture both interactions between species as well as a factor (e.g., abundance, movement, physiology) helping to determine those interactions.

Ecology has always championed the notion that complexity should never be embraced for complexity's sake alone, except when the tools to face the challenge are available (Green et al., 2005) and the alternative is an unpalatable sacrifice of biological realism (May, 1972). With this in mind, the EMN framework represents an important step towards capturing the true complexity of ecological communities. The methodological tools to analyse these networks are already established (Boccaletti et al., 2014; Kivelä et al., 2014); as a technical advance, the power of multilayer networks is therefore clear. However, a methodological framework to describe and study EMNs is not enough to satisfy the all-important question, "so what?" While all methodological innovations require use to be broken in and prove their value, it is still unclear how describing EMNs will improve our understanding of ecological communities. To guide this process, we identify several considerations for the empirical implementation of EMNs and outline a series of research areas that we believe will most immediately benefit. 
(a) Motifs across layers

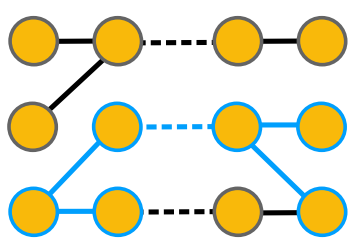

(d) Adaptation in space

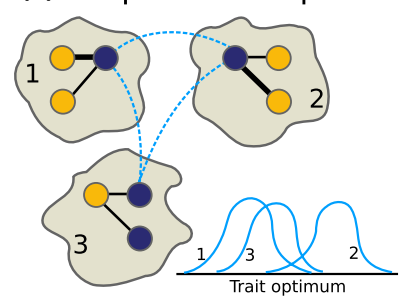

(b) Niche partitioning

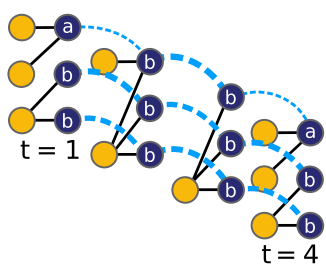

(e) Ontogenetic EMNs

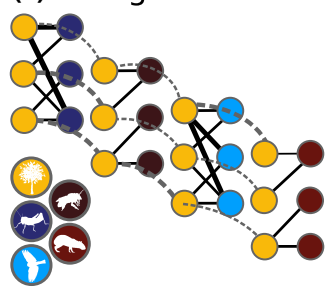

(c) Percolation in EMNs

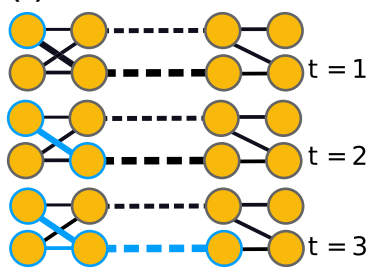

FIGURE 1 Examples where we expect EMNs to be innovative for community ecology. (a) How interaction patterns within layers are connected between them can give definition to the interconnectedness of networks across interaction types. Here, 3-species motifs are highlighted (blue outline) as one pattern worth investigating. (b) Identifying groups of species across an EMN provides evidence for the consistency of niche partitioning. Here, letters represent hypothetical groups based on inter- and intra-layer edges. (c) Percolation across EMNs can be used to study how ecosystems will respond to perturbation. Blue outlining marks the trajectory of percolation here and a key question is how inter-layer edges representing ecological processes will transmit perturbations. (d) Three habitat patches are represented, and populations of one species (upper dark blue circle) are connected between them. Different interaction patterns between layers determine functional-trait optima, and inter-layer edges may be key to adaptation across the system. (e) A multiplex network of seasonal attachment by a tree population to different animal guilds is represented. Interaction patterns within and across layers could be used to determine individual fitness outcomes

\section{2 | INTER-LAYER EDGES: WHY, WHEN, HOW}

Inter-layer edges connect nodes between layers (usually these are between a species and its representation in each layer but see Gracia-Lázaro et al., 2018), and they are the key component that differentiates multilayer networks from previous approaches to ecological networks. Incorporating inter-layer edges into ecological networks allows ecological processes that influence interaction patterns to be incorporated into network structure creating a more complete description of community dynamics. These edges may represent a myriad of processes, and their definition will depend on the specifics of the system and questions at hand. Because inter-layer edges are essential to a multilayer approach, we discuss three main considerations for their use: (a) Do inter-layer edges add necessary information? (b) Can inter-layer edges have the same weight across the network or should they be specific to pairs of nodes? (c) How can inter-layer edges be quantified?

\subsection{Why use inter-layer edges and EMNs?}

In many cases, inter-layer edges will represent ecological processes familiar to community ecologists. For example, in spatial EMNs (Figure 1d), inter-layer edges can represent movement between layers, similar to meta-communities; in temporal EMNs (e.g., Costa et al., 2018; Figure 1c), they can represent changes in abundance, echoing the interplay between abundance and foraging inherent in population and functional response theories; in pathogen systems, they can represent temporal genetic changes, similar to phylogenetic trees in phylodynamics (Pilosof et al., 2018). That each of the processes represented by inter-layer edges already have theoretical frameworks built around them begs the question: why study them with an EMN approach?

First, we already know that, for example, dispersal between sites can maintain local resource populations-and therefore consumer-resource interactions-in areas where they would otherwise become locally extinct (Rosenheim, 2001), or that changes in resource species' density alters consumer behaviour (Holling, 1959). EMNs provide a way to integrate existing knowledge of this sort into the structure of ecological networks for a more holistic study of species interactions. Second, by combining species interactions (intra-layer edges) with existing knowledge, as inter-layer edges, the types of questions that will be addressed with ecological networks become more salient. With a set of networks distributed in space, the logical question might be to understand how these networks differ based on their proximity. Instead if those networks are studied as a single spatial EMN, for which proximity-based effects are structurally explicit, then the questions that are most intuitive to ask become those that pertain to population and community dynamics, biogeography and ecosystem management (Figures 1 and 2). A sharper focus on these areas can improve the ability of network ecology to speak to fundamental questions around biodiversity maintenance and their applied counterparts in conservation (Box 1). 


\subsection{When do inter-layer edges need to be quantified?}

Inter-layer edges may take several forms from none and uniform edge weights (including binary weights) to non-uniform edges. EMNs with no inter-layer edges are a set of independent networks (identical to previous approaches in network ecology that have tried to assess spatial or temporal variation; e.g., Poisot, Canard, Mouillot, Mouquet, \& Gravel, 2012; Cirtwill, Roslin, Rasmussen, Olesen, \& Stouffer, 2018). When EMNs have uniform inter-layer edges (i.e., all edges between layer have the same weight), they assume that all connections between all layers have the same effect and inter-layer edges effectively describe the assumed dependence of layers on each other. Finally, if interlayer edges are non-uniform (i.e., each link between nodes in different layers will be distinct), they represent the actual or estimated linkage between two nodes and add substantial resolution to the network. EMNs with different types of inter-layer edges can be used to ask different questions (Table 1) even though the most complete description of the community will require non-uniform inter-layer edges.

When inter-layer edges have been used, they are often uniform (Kéfi, Miele, Wieters, Navarrete, \& Berlow, 2016; Timóteo, Correia, Rodríguez-Echeverría, Freitas, \& Heleno, 2018). The strength of uniform inter-layer edges is an assumption about the interdependence of layers. If inter-layer edge strength is much greater than intra-layer strengths, inter-layer processes dominate the community and determine structural properties and vice versa (Pilosof et al., 2017). In Gorongosa National Park, seed-dispersal modules become habitat-specific when the inter-layer weighting is small relative to intralayer weights; however, when inter-layer weightings were large, landscape-level groupings of plant-disperser interactions emerged (Timóteo et al., 2018). These results naturally suggest that when layers (in this case, habitats) are considered to be distinct ecological units, species interactions become grouped by those, often arbitrary, borders. However, when a more continuous view is taken-as is done in Timóteo et al., 2018 when inter-layer edges become large relative to intra-layer edges-a holistic image of those interactions across the landscape, community or time span emerges.

Shifting from uniform to non-uniform inter-layer edges in EMNs can provide more detailed information on the processes that operate between layers and their role in community structure. In a spatial EMN in which inter-layer edges represent inter-patch relationships (e.g., species-specific movement or predation risk differences between patches; Figure 1d), non-uniform inter-layer edges will explicitly incorporate variation in inter-patch movement between species and populations. This variation is crucial because it will eventually affect within-patch population dynamics (Rosenheim, 2001). Likewise in temporal networks constructed for highly seasonal ecosystems, inter-layer edges representing fluctuations in species' abundance are bound to be non-uniform because uniform inter-layer edges will ignore the species-specific responses to seasonality and therefore distort conclusions about how temporal resource competition or availability structures the community (Costa et al., 2018). Accordingly, if inter-layer edges linking plant species across seasons are uniform they assume that the effect of season on plant biomass is equivalent across species despite the fact that annuals may switch from low to high biomass while a woody perennial maintains biomass between seasons (e.g., Singh \& Yadava, 1974).

\subsection{How can inter-layer edges be quantified?}

Measuring inter-layer edges will often be straightforward given ecology's extensive empirical toolbox. Community sampling tools such as camera-trapping grids (and other community sampling methods) can be particularly useful for estimating species' relative abundance given their ability to yield data for large numbers of species with relatively little time investment. Recent developments (Norouzzadeh et al., 2018) mean that extracting occupancy measures from cameratrap data represents a low-cost and low-intensity way to estimate inter-layer edges for both spatial and temporal EMNs. Similarly, GPS tags, population genetic monitoring and mark-recapture techniques can estimate specieswise connectivity between layers in spatial EMNs (Table 1). In temporal EMNs, where time points are modelled as layers, species' abundance and biomass are modelled as inter-layer edges and can be estimated with a variety of standard approaches (Table 1).

Non-uniform inter-layer edges in multiplex EMNs are less obvious, but an intuitive representation would be the effect of one interaction type on another. These might be estimated by applying specific natural history knowledge of the species that connect layers. For example, a multiplex EMN where one layer represents predator-prey interactions and another represents plant-herbivore interactions could have inter-layer edges that describe how the landscape of fear alters prey/herbivore habitat use. A landscapeof-fear-derived measure would capture the way that predation on herbivores alters their foraging patterns thereby linking herbivory patterns to predation interactions. Similarly, in the case of dispersers and predators of seeds, inter-layer edges representing interference between these two guilds might be measured as the efficiency of fruit removal by dispersers, which would deny foraging opportunities for seed predators locally.

If empirical quantification is impossible, inter-layer edges may also be inferred by combining natural history and ecological theory. Morphological traits are intricately linked to ecological network structure, and this is particularly true for body size (Brose, Williams, \& Martinez, 2006). In addition, larger bodied species tend to have larger home ranges and dispersal ability (Bowman, Jaeger, \& Fahrig, 2002; Lindstedt, Miller, \& Buskirk, 1986). Therefore, in spatial EMNs, combining body size with distance between locations (layers) could be used to generate species- and layer-specific dispersal potentials.

It is also possible to infer inter-layer edges based on intra-layer edges. Neighborhood flow coupling-where inter-layer weights are designated based on the similarity of a node's intra-layer edges between two layers-provides one approach to do so (Aslak, Rosvall, \& Lehmann, 2018) and would be appropriate for temporal and spatial EMNs. For example, if a plant is visited by the same pollinators in two consecutive years, this approach would generate a strong inter-layer 


\section{Box 1 Multilayer networks for ecosystem management}

The impetus for an EMN approach to ecology stems from the idea that they can capture a greater portion of ecosystem complexity (Fontaine et al., 2011; Pilosof et al., 2017), and capturing such complexity is particularly necessary for ecosystem management. Ecological multilayer networks offer several opportunities for applied ecology. More than a decade ago, the Millennium Ecosystem Assessment identified four key anthropogenic drivers of global change: climate change, nutrient pollution, invasive species and land-use change (Millennium Ecosystem Assessment, 2005). Here, we highlight potential uses of an EMN approach to ecosystem management by exploring examples pertinent to each of these four drivers.

The warming of the world's oceans is of particular concern for coral reefs and the ecological associations (Sale, 2013) that coral-zooxanthellae symbiosis supports. A multiplex EMN-where layers are interaction types such as coral-zooxanthellae association, coralalgae competition, herbivory and cleaning mutualisms while inter-layer edges represent the dependence of interaction types on each other-could be used to assess how damage to coral-zooxanthellae associations might cascade to the rest of the community (Figure 2a). Nutrient pollution can alter plant communities (Hautier, Niklaus, \& Hector, 2009) and potentially the pollinator, herbivore and predator-prey webs they support. Multiplex EMNs founded upon plant associational interactions could be used to examine how nutrientdriven changes in plant community composition will predict ecosystem service provisioning by animals based on percolation from the plant-plant layer to higher trophic layers (Figure 2b). The proliferation of non-native species is reality in a globalised world, and establishment is influenced by trophic interactions (Maron \& Vilà, 2001; Salo, Korpimäki, Banks, Nordström, \& Dickman, 2007). An invasion biology application of EMNs might take a meta-community approach and consist of habitat-fragment layers, inter-layer edges encoding species-specific dispersal between fragments, and be used to forecast native resistance and define management actions (Figure 2c). Land conversion for anthropogenic purposes is rampant (Ramankutty, Evan, Monfreda, \& Foley, 2008) and can profoundly impact species' abundance and ecological interactions (Ponisio et al., 2017). A temporal EMN would allow the dynamics of community decomposition or assembly under land-use change to be precisely identified. Each layer might be a food web at a certain point in time with inter-layer edges representing change in abundance and will be useful for determining how ecological community outcomes are linked to land-use change (Figure $2 \mathrm{~d}$ ).

(a) Climate impact on reef ecosystems

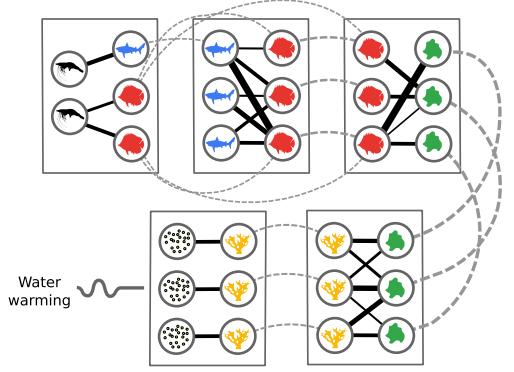

(b) Nutrient-based community turnover

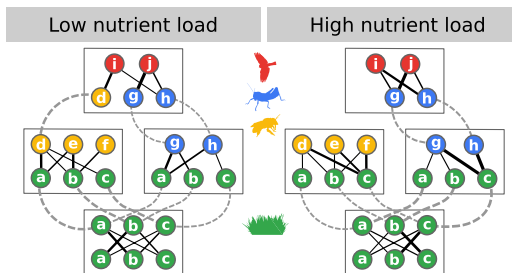

(c) Spatial refugia for native species

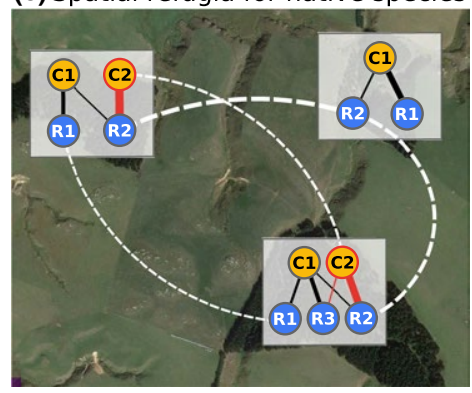

(d) Food web assembly and land use

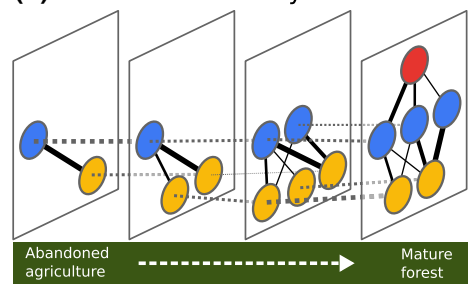

FIGURE 2 EMNs and global change ecology. (a) A multiplex EMN of a coral reef. Intra-layer edges describe ecological interactions such as competition between coral and algae. Inter-layer edges represent the per species fitness effects of one interaction type on the other. (b) Two hypothetical multiplex EMNs for low- and high-nutrient loads show how changes in nutrient load may cascade upwards from plant associations to higher webs (colours represent guilds; red: insectivores, yellow: pollinators, blue: herbivores, green: plants). (c) Food webs for three habitat fragments with dispersal as inter-layer edges (white dotted lines). In this example, C2 is invasive and specialises on R2. Inter-layer edges between patches allow R2 to persist. Map data: Google, DigitalGlobe. (d) Food web assembly as land-use changes from agriculture to forest. Circles are different species, and colour indicates their trophic level. Interlayer edges depict change in abundance between time points 
TAB LE 1 Quantifying inter-layer edges in multilayer networks

\begin{tabular}{|c|c|c|c|}
\hline Inter-layer edge & Network type & Significance & Quantifying edges \\
\hline Dispersal between patches & Spatial EMN & $\begin{array}{l}\text { Movement of individuals between patches } \\
\text { creates a correlation in the structure of } \\
\text { those layers. Inter-layer edges weighted by } \\
\text { dispersal recognise movement as a } \\
\text { determinant of interspecific interaction } \\
\text { patterns }\end{array}$ & $\begin{array}{l}\text { GPS tracking of individuals, camera } \\
\text { trapping in dispersal corridors, population } \\
\text { genetic monitoring of patches or } \\
\text { mark-recapture }\end{array}$ \\
\hline Difference in abundance & $\begin{array}{l}\text { Spatial and } \\
\text { temporal EMNs }\end{array}$ & $\begin{array}{l}\text { Abundance influences interspecific interac- } \\
\text { tions. Inter-layer edges representing } \\
\text { abundance allow the EMN to capture } \\
\text { population and community trends } \\
\text { simultaneously }\end{array}$ & $\begin{array}{l}\text { Community monitoring within layers. For } \\
\text { animals, trapping grids, eDNA monitoring, } \\
\text { point counts or mark-recapture. For } \\
\text { plants, quadrat- or transect-based surveys } \\
\text { of biomass and eDNA monitoring }\end{array}$ \\
\hline $\begin{array}{l}\text { Dependency of species } \\
\text { interaction types }\end{array}$ & Multiplex EMN & $\begin{array}{l}\text { Species' participation in distinct interaction } \\
\text { types and their effect on each other } \\
\text { determine network structure }\end{array}$ & $\begin{array}{l}\text { Experimental determination of fitness } \\
\text { differences in the presence of one or both } \\
\text { interactions. Proxies for fitness differ- } \\
\text { ences are more realistic }\end{array}$ \\
\hline $\begin{array}{l}\text { Change in energetic } \\
\text { requirements }\end{array}$ & $\begin{array}{l}\text { Individual-based } \\
\text { temporal EMN }\end{array}$ & $\begin{array}{l}\text { Energetic requirements can change through } \\
\text { time. Pregnancy and lactation can double an } \\
\text { animal's energetic requirements and alter } \\
\text { foraging decisions }\end{array}$ & $\begin{array}{l}\text { Expected change in energetic require- } \\
\text { ments. May be quantified through a } \\
\text { combination of individual physiological } \\
\text { status and literature-derived values for } \\
\text { the effect of changing state }\end{array}$ \\
\hline $\begin{array}{l}\text { Changing predation/ } \\
\text { parasitism risk. }\end{array}$ & $\begin{array}{l}\text { Spatial and } \\
\text { temporal EMNs }\end{array}$ & $\begin{array}{l}\text { Risk can change through space and time. } \\
\text { Inter-layer edges capturing differences in } \\
\text { risk between layers can link direct and } \\
\text { trait-mediated consumer effects }\end{array}$ & $\begin{array}{l}\text { Environmental monitoring of parasite } \\
\text { transmission risk and predator } \\
\text { populations }\end{array}$ \\
\hline Spatial subsidies & Spatial EMN & $\begin{array}{l}\text { Ecosystems and communities do not exist in } \\
\text { isolation, and inter-layer edges can } \\
\text { represent the flow of nutrients from one } \\
\text { patch to the other }\end{array}$ & $\begin{array}{l}\text { Environmental monitoring between } \\
\text { patches }\end{array}$ \\
\hline
\end{tabular}

link between the plant species in those two layers likely implying maintenance of abundance and pollination service between years. A similar approach could infer inter-layer edges representing the change in abundance of species $i$ between a layer at time $t$ and a layer at time $t+1$ based on the net effect of i's intra-layer edges at $t$. Inter-layer edges with inferred weights should not remove the incentive to measure inter-layer dependencies but may provide more information to an analysis than uniform inter-layer edges because, even if they only capture coarse differences between species (e.g., evergreen vs. deciduous), they will bring greater natural history into the structure of EMNs.

\subsection{Additional considerations when describing, quantifying and collating EMNs}

The quantification of species interactions is a much-discussed facet of community ecology (Novak et al., 2016). In an EMN scenario, where different interaction types are represented, an additional challenge is to quantify interactions in a common currency and has thus far proven difficult (García-Callejas et al., 2018). The per capita effect of one species on the other is key to theory that underlies food webs (May, 1972) and becomes the gold standard since it is not specific to an interaction type (Novak et al., 2016). Several approaches exist for quantifying interactions in this way, and these need not always be experimental (Bimler, Stouffer, Lai, \& Mayfield, 2018; Lanuza, Bartomeus, \& Godoy, 2018). Given the importance of per capita effects in ecological theory, it is important that these emerging approaches are built on to help generate accurate species interaction data because, with increasing richness, experimental approaches quickly become impractical; however, there is still substantial work to be done (Barner, Coblentz, Hacker, \& Menge, 2018).

Alternatively, interaction frequency has been used as a proxy for per capita interaction strength (Vázquez, Morris, \& Jordano, 2005). However, it is unclear how comparable the frequencies of two interaction types are to each other. For example, it is unclear how a bird eating five fruits is comparable to five parasites being present in that bird's gastrointestinal tract. While transforming raw frequencies to relative frequencies would be one way to make these numbers more comparable, a potential common currency for species interactions-relative read abundance (RRA)-goes a step further. RRA quantifies interactions from DNA meta-barcoding studies and is the proportion of a sample, perhaps pollen on a bee's legs or plant material in an ungulate's dung, assigned to each resource taxon (Kartzinel et al., 2015). Because read abundance is a count of DNA barcodes belonging to each taxon, RRA counts ecological interactions in the same way regardless of interaction type. DNA meta-barcoding is particularly relevant to trophic interactions and even 
difficult-to-describe interactions such as intra-guild predation can feasibly be studied (Toju \& Baba, 2018). Non-trophic interactions require additional study to identify how they might be estimated with DNA barcoding. For example, below-ground plant species' RRA (Hiiesalu et al., 2012) should be examined in relation to plant-plant competition. The wide-ranging applications of DNA meta-barcoding to species interactions, declining cost and consistent approach to quantifying interactions make RRA a potential solution to empirically constructing the intralayer edges of multiplex EMNs in a standardised way.

Even when harnessing technological advances to estimate inter- and intra-layer edges, substantial boots-on-the-ground effort is required to collect EMNs. Fortunately, several solutions to the problem of big data collection exist. Foremost, large-scale collaborations represent an ideal facilitator of such endeavours. Ecological questions of comparable complexity have been embraced through massive collaborative efforts such as the Nutrient Network experiment and macroecological working groups. It is also important that EMNs are collated in open-access databases since ecological network databases have been crucial to the identification of patterns in ecological networks (e.g., Olesen, Bascompte, Dupont, \& Jordano, 2007; Hutchinson, Cagua, \& Stouffer, 2017). The existence of platforms that curate and standardise data such as mangal (http://mangal.io/; Poisot et al., 2016) and globi (https://www.globalbioticinteractions.org/; Poelen, Simons, \& Mungall, 2014) will streamline the collation and curation of EMNs as well as facilitate their use in identifying patterns in community organisation.

\section{3 | PUTTING EMNS TO WORK IN COMMUNITY ECOLOGY}

Now that we have described the considerations involved in obtaining EMN data, we turn to several areas of active research in community ecology where the application of EMNs can lead to conceptual advances. For each area below, we briefly describe a few relevant questions and hypotheses an EMN approach can address and the ways in which it may be implemented.

\subsection{The structure of species interaction networks}

The search for non-random structural patterns in ecological networks has generated some of the clearest evidence for consistency in the organisation of ecological communities. Insights into niche partitioning (Beckerman, Petchey, \& Warren, 2006), forbidden interactions (Olesen et al., 2011) and sampling of interactions (Jordano, 2016) have been garnered from the observation that rarely more than $30 \%-40 \%$ of possible interactions are realised in food webs (Fortuna et al., 2010). In EMNs, exploring basic structural properties and, in particular, the linkage patterns between inter-layer edges and intra-layer structure deserves attention (Table 1). One area of particular interest with respect to network structural patterns is the way that plants are connected to the multitude of primary consumer guilds that they support. Initial findings suggest that plants do connect herbivores and pollinators in non-random ways (Sauve, Thébault, Pocock, \& Fontaine, 2016) and this should be expanded to other primary consumer groups too.

The identification of structural patterns in EMNs requires null models-which evaluate empirical structure in comparison with random expectation-within which to assess the consistency of observed patterns. In a EMN case, a null model can focus on the placement of inter-layer edges and the ordering of layers as well as intra-layer edges (Bassett, Wymbs, PorterMA, Carlson, \& Grafton, 2011). Null models addressing particular EMN components can be particularly useful in isolating the mechanisms producing structural patterns (Bassett et al., 2011). The value of shuffling intra- and interlayer edges at the same time deserves attention as well as the comparison of shuffling inter-layer edge weights vs. edge presence.

The development of null models for EMNs and the study of overall network metrics, however, should focus on answering specific ecological questions. To this end, there are some computational tools that could provide valuable insights (see Pilosof et al., 2017 for a list of metrics). In particular, the study of network motifs-the suite of $n$-node interaction patterns within networks-has already proven to be a useful tool in characterising the structural roles that species play in networks (Bramon Mora, Gravel, Gilarranz, Poisot, \& Stouffer, 2018) and could provide an interesting perspective on how interaction patterns are linked across layers (Figure 1a). Studying which motifs are linked to which across layers could shed light on the dynamics of the interaction patterns and the consistency of species' roles (as defined by motif membership) across layers. We predict that strong inter-layer edge weights will be associated with species' role consistency across layers because stronger inter-layer edges will tend to represent the relatedness or dependency of the focal species' interactions in each layer.

\subsection{Niche partitioning across time and space}

Resource partitioning is central to favoured explanations of diversity in ecological communities (MacArthur \& Levins, 1967) and is often observed (McDonald, 2002). Ecological networks have been used to examine niche partitioning for diverse communities (Blüthgen, Fründ, Vázquez, \& Menzel, 2008), but many network datasets comprise a small spatial or temporal window. Characterising resource partitioning within communities in a spatial or temporal EMN will provide an explicit link to models of resource competition and generate a comprehensive picture of specialisation that simultaneously considers variation across space and time as well as the process encoded in the inter-layer edges which will usually be related to population change.

Large mammalian herbivores in Africa are an illustrative example since their resource use has been studied thoroughly. These species tend to be either grazers (grass feeders) or browsers (woody-plant feeders), with few species being mixed feeders (Hempson, Archibald, \& Bond, 2015). However, plant communities 
in savanna ecosystems are highly seasonal and it is unclear how strictly grazers and browsers separate. A temporal EMN would allow novel access to this question if layers represent mammalplant herbivory networks at different times of year and inter-layer edges represent changes in population density across the year (Figure 1b). We expect the group structure of this EMN would be more inclusive in periods where resources are abundant and dissipates to more traditional grazer-browser separation during low resource availability. These results, which describe the behavioural plasticity in large mammal-herbivore foraging, would reveal the intensity of resource limitation on such communities and their ability to rewire their diets to cope.

\section{3 | Network stability}

Central to the adoption of a network approach in ecology was the question of whether diverse systems of interacting species can be stable (May, 1972). In monolayer networks, stability is typically studied as resilience (Thébault \& Fontaine, 2010), robustness (Dunne \& Williams, 2009) or local stability (Allesina \& Pascual, 2008). EMNs provide a more integrated view of the communities studied, and alternate methods must be developed for EMN stability analysis (but see Reis et al., 2014; Danziger, Shekhtman, Bashan, Berezin, \& Havlin, 2016). In a recent meta-community study-which studied a spatial EMN in all but name-May's original formulation of local stability was expanded to include an additional matrix of dispersal rates, finding that dispersal can increase stability of meta-ecosystems (Gravel, Massol, \& Leibold, 2016). These results highlight the crossover between existing theory of ecological meta-communities and EMNs while also emphasising the importance of transitioning to larger scale approaches to inform ecosystem management decisions. Excitingly, the approach of Gravel et al. (2016) is transferable to other types of multilayer networks if their dispersal matrix is generalised to represent any inter-layer edge.

The spread of disturbances-percolation-through ecological networks has also generated a vibrant literature, particularly in the case of extinction cascades (Dunne \& Williams, 2009). Percolation is even more pertinent in multilayer networks since percolation between layers could be particularly relevant to the ecosystem management scenarios that EMNs capture. Several models exist from statistical physics of interdependent networks (Buldyrev, Parshani, Paul, Stanley, \& Havlin, 2010; Huang, Gao, Buldyrev, Havlin, \& Stanley, 2011; Liu, Eisenberg, Seager, \& Lai, 2018), and others are needed to understand how percolation proceeds when inter-layer edges represent different processes or are non-uniform (Figure 1c). Pressingly, different parts of ecosystems can be represented by EMNs that could help identify key edges that transmit perturbations and provide specific management directives. Since modules buffer percolation in monolayers networks (Gaiarsa and Guimarães, 2018), we expect that extinction cascades within layers of, for example, a spatial EMN will remain localised if sink populations are affected, or if source populations are affected extinction will percolate across layers.

\section{4 | Interaction-driven adaptation}

Understanding how species interactions drive adaptation in their participants is increasingly important (Galetti et al., 2013). Direct and indirect interactions in ecological networks can have substantial effects on co-evolutionary outcomes (Guimarães, Pires, Jordano, Bascompte, \& Thompson, 2017). An extension of these findings is to explore how population-level adaptation occurs across a multiplex EMN when the species being studied are involved in a variety of interaction types concurrently (Fontaine et al., 2011).

The close matching between plant floral traits and the bill morphology of flower-visiting birds such as hummingbirds (Cotton, 1998) reflects the importance of their association. However, hummingbirds also have predators (Stiles, 1978), parasites (Moens et al., 2016) and competitors (Laverty \& Plowright, 1985). Representing different aspects of hummingbird ecology as a multiplex EMN allows these interactions and their selective pressures to be studied together. We expect that an adaptive interplay between flower visitation and competition layers will occur on a multiplex EMN due to strong inter-layer edges (visitation can determine competition and vice versa) that leads to selection on bill morphology and adaptive rewiring of intra-layer edges and their weights in both layers.

A spatial setting is also pertinent to trait adaptation on EMNs. The geographic mosaic of co-evolution (Thompson, 2005) describes co-evolution occurring across a landscape of patches and a spatialEMN framework is an ideal tool to study these patches as an integrated whole. Inter-layer edges representing either trait similarity between populations in different layers or gene flow between layers would generate a description of the geographic mosaic that would then allow models of trait adaptation to make predictions about coevolution at a landscape level (Figure 1d).

\section{5 | Predicting interactions from species traits}

Trait-based ecology has made explicit the link between species' morphology and ecological processes (McGill, Enquist, Weiher, \& Westoby, 2006), and has been successfully applied in reducing the dimensionality of complex ecological networks (Eklöf et al., 2013; Laigle et al., 2018). Extrapolating these results, a suite of traits could be leveraged to predict multiplex EMNs. For example, plants face a strong trade-off between competitive ability and herbivory defence (Kempel, Schädler, Chrobock, Fischer, \& Kleunen, 2011) and plant secondary compounds influence both herbivory and pollination (Georgelin \& Loeuille, 2016). Initial explorations suggest that these correlations may underlie entire communities (Sauve et al., 2016).

While using several traits to predict multiplex EMNs across the web of life is likely within reach, it is also possible that there are individual "skeleton key" traits that can predict multiplex EMN structure alone. One promising avenue is the nutritional value of resources. Traits representing some measure of nutritional value-be they body size, the leaf economic spectrum or biochemical composition-have been consistently powerful for community ecology. We expect that the description of a standardised nutritional quality trait will predict 
multiplex network structure better than existing models. Such a trait would be a massive coup for community ecology because it would capture a common feature underpinning the organisation of ecological communities.

\subsection{Ontogenetic shifts in species interactions}

For many species, the suite of potential ecological interaction partners is not the same throughout an individual's life. Predators will eat bigger prey as they grow, parasites often infect different hosts throughout their life cycle, and lepidopterans switch from being herbivores to mutualists. Importantly, incorporating ontogeny into food webs yields networks less robust to extinctions (Rudolf \& Lafferty, 2011). In a scenario as distinct as lepidopteran metamorphosis, considering the caterpillar-plant and butterfly-plant associations together as layers of multiplex network could be used to explore their impact on plant communities across the life cycle of these insects (Astegiano, Altermatt, \& Massol, 2017).

Individual-based networks, where the same individual "builds" different ecological interactions with multiple partners through time, are also a natural place for ontogenetic EMNs to be explored. A recent study demonstrated how nurse plant age is positively related to the nestedness of their associational plant networks (Losapio, Pugnaire, O'Brien, \& Schöb, 2018). Similarly, trees in a population interact sequentially across the growing season with folivores, pollinators, pre-dispersal seed predators, frugivores, postdispersal seed predators. Multiplex EMNs could be used to study this succession where inter-layer edges represent the proportion of individual investment progressing to the next stage of reproduction (e.g., proportion of flowers pollinated, proportion of seeds dispersed). We predict that the group structure in these multiplex EMNs (Figure 1e) will be related to individual fitness and may be used to forecast the generational shifts in allele frequencies of the population. Moreover, we expect that intra-layer edge structures will be predictive of inter-layer weights (an individual visited in the greatest frequency and by the greatest diversity of pollinators will contribute a larger investment to the next stage of reproduction).

\section{4 | CONCLUSIONS}

Ecological communities and meta-communities are complex systems that harbour immense diversity. Through time, methodological developments in ecology have allowed increasing amounts of that diversity to be described and analysed simultaneously. Ecological networks representing static images of ecological communities have yielded important findings on many aspects of community organisation. Multilayer networks appear to be the logical next step in that expanding toolbox because EMNs make it possible for additional realism-such as population dynamics across space and time as well as multiple types of species interactions-to be built into the structure of species interaction networks.

Of course, increasing the realism of already data-rich analyses necessitates greater empirical effort to generate interaction networks. Fortunately, the possibilities inherent in emerging data collection technologies, such as DNA meta-barcoding, camera trapping and remote sensing, combined with tried-and-tested field observations and increasing accessibility of ecological data mean that leveraging EMNs to tackle fundamental questions in community ecology is an achievable ambition. Ultimately, EMNs have the potential to provide the larger scale and detailed descriptions of ecological communities that may help address historical laments in community ecology (Lawton, 1999; Paine, 1988). Looking forward, it is impossible to determine the impact that EMNs will have on community ecology, but the prospects are bright. All that remains is for boots to hit the ground.

\section{ACKNOWLEDGEMENTS}

The authors would like to thank the Editor, Associate Editor and two anonymous reviewers for constructive and insightful comments on the manuscript. M.C.H. acknowledges an Inspire Foundation (Christchurch, New Zealand) grant and funding from Princeton University. D.B.S. and B.B.M. acknowledge a Rutherford Discovery Fellowship, from Government funding administered by the Royal Society of New Zealand (to D.B.S.). A.K.B. and S.P. acknowledge the Postdoctoral Program in Complexity Science-Complex Systems Fellowship Award administered by the James S. McDonnell Foundation's 21st Century Science Initiative. SK was supported by the ARSENIC project, grant no. 14-CE02-0012, awarded by the French National Research Agency. P.J. acknowledges grants CGL2017-82847-P and Severo Ochoa Excellence Award from the Ministerio de Economía y Competitividad (SEV-2012-0262), Spain.

\section{AUTHORS' CONTRIBUTIONS}

All authors contributed to the formulation, writing and editing of the manuscript.

\section{DATA ACCESSIBILITY}

There are no data associated with this manuscript.

\section{ORCID}

Matthew C. Hutchinson (iD https://orcid.org/0000-0002-2423-4026

Pedro Jordano (iD https://orcid.org/0000-0003-2142-9116

Daniel B. Stouffer (ID) https://orcid.org/0000-0001-9436-9674

\section{REFERENCES}

Allesina, S., \& Pascual, M. (2008). Network structure, predator-prey modules, and stability in large food webs. Theoretical Ecology, 1, 55-64.

Aslak, U., Rosvall, M., \& Lehmann, S. (2018). Constrained information flows in temporal networks reveal intermittent communities. Physical Review E, 97, 062312. https://doi.org/10.1103/ PhysRevE.97.062312

Astegiano, J., Altermatt, F., \& Massol, F. (2017). Disentangling the costructure of multilayer interaction networks: Degree distribution 
and module composition in two-layer bipartite networks. Scientific Reports, 7, 15465. https://doi.org/10.1038/s41598-017-15811-w

Barabási, A. L. (2011). The network takeover. Nature Physics, 8, 14.

Barner, A. K., Coblentz, K. E., Hacker, S. D., \& Menge, B. A. (2018). Fundamental contradictions among observational and experimental estimates of non-trophic species interactions. Ecology, 99, 557-566. https://doi.org/10.1002/ecy.2133

Bascompte, J., \& Jordano, P. (2013). Mutualistic networks (Vol. 70). Princeton, NJ: Princeton University Press.

Bassett, D. S., Wymbs, N. F., PorterMA, M. P. J., Carlson, J. M., \& Grafton, S. T. (2011). Dynamic reconfiguration of human brain networks during learning. Proceedings of the National Academy of Sciences of the United States of America, 108, 7641-7646. https://doi.org/10.1073/ pnas.1018985108

Beckerman, A. P., Petchey, O. L., \& Warren, P. H. (2006). Foraging biology predicts food web complexity. Proceedings of the National Academy of Sciences of the United States of America, 103, 13745-13749. https:// doi.org/10.1073/pnas.0603039103

Bimler, M. D., Stouffer, D. B., Lai, H. R., \& Mayfield, M. M. (2018). Accurate predictions of coexistence in natural systems require the inclusion of facilitative interactions and environmental dependency. Journal of Ecology, 106, 1839-1852. https://doi.org/10.1111/1365-2745.13030

Blüthgen, N., Fründ, J., Vázquez, D. P., \& Menzel, F. (2008). What do interaction network metrics tell us about specialization and biological traits. Ecology, 89, 3387-3399. https://doi.org/10.1890/07-2121.1

Boccaletti, S., Bianconi, G., Criado, R., Del Genio, C. I., Gómez-Gardenes, J., Romance, M., ... Zanin, M. (2014). The structure and dynamics of multilayer networks. Physics Reports, 544, 1-122. https://doi. org/10.1016/j.physrep.2014.07.001

Bowman, J., Jaeger, J. A., \& Fahrig, L. (2002). Dispersal distance of mammals is proportional to home range size. Ecology, 83, 2049-2055. https:// doi.org/10.1890/0012-9658(2002)083[2049:DDOMIP]2.0.CO;2

Bramon Mora, B., Gravel, D., Gilarranz, L. J., Poisot, T., \& Stouffer, D. B. (2018). Identifying a common backbone of interactions underlying food webs from different ecosystems. Nature Communications, 9, 2603. https://doi.org/10.1038/s41467-018-05056-0

Brose, U., Williams, R. J., \& Martinez, N. D. (2006). Allometric scaling enhances stability in complex food webs. Ecology Letters, 9, 1228-1236. https://doi.org/10.1111/j.1461-0248.2006.00978.x

Buldyrev, S. V., Parshani, R., Paul, G., Stanley, H. E., \& Havlin, S. (2010). Catastrophic cascade of failures in interdependent networks. Nature, 464, 1025. https://doi.org/10.1038/nature08932

Cirtwill, A. R., Roslin, T., Rasmussen, C., Olesen, J. M., \& Stouffer, D. B. (2018). Between-year changes in community composition shape species roles in an arctic plant-pollinator network. Oikos, 127, 11631176. https://doi.org/10.1111/oik.05074

Costa, J. M., Ramos, J. A., Timóteo, S., da Silva, L. P., Ceia, R. S., \& Heleno, R. H. (2018). Species activity promote the stability of fruit-frugivore interactions across a five-year multilayer network. bioRxiv, 421941.

Cotton, P. A. (1998). Coevolution in an amazonian hummingbird-plant community. Ibis, 140, 639-646. https://doi.org/10.1111/j.1474919X.1998.tb04709.x

Danziger, M. M., Shekhtman, L. M., Bashan, A., Berezin, Y., \& Havlin, S. (2016). Vulnerability of interdependent networks and networks of networks. In A. Garas (Ed.), Interconnected networks (pp. 79-99). Basel, Switzerland: Springer

Dunne, J. A., \& Williams, R. J. (2009). Cascading extinctions and community collapse in model food webs. Philosophical Transactions of the Royal Society B: Biological Sciences, 364, 1711-1723. https://doi. org/10.1098/rstb.2008.0219

Egerton, F. N. (2012). Roots of ecology: Antiquity to Haeckel. Berkeley, CA: University of California Press.

Eklöf, A., Jacob, U., Kopp, J., Bosch, J., Castro-Urgal, R., Chacoff, N. P., ... Allesina, S. (2013). The dimensionality of ecological networks. Ecology Letters, 16, 577-583. https://doi.org/10.1111/ele.12081
Fontaine, C., Guimarães, P. R., Kéfi, S., Loeuille, N., Memmott, J., van Der Putten, W. H., ... Thébault, E. (2011). The ecological and evolutionary implications of merging different types of networks. Ecology Letters, 14, 1170-1181. https://doi.org/10.1111/j.1461-0248.2011.01688.x

Fortuna, M. A., Stouffer, D. B., Olesen, J. M., Jordano, P., Mouillot, D., Krasnov, B. R., ... Bascompte, J. (2010). Nestedness versus modularity in ecological networks: Two sides of the same coin? Journal of Animal Ecology, 79, 811-817. https://doi. org/10.1111/j.1365-2656.2010.01688.x

Gaiarsa, M. P., \& Guimarães, P. Jr (2018). Interaction strength promote robustness in mutualistic networks against cascading effects. Scientific Reports. https://doi.org/10.1038/s41598-018-35803-8

Galetti, M., Guevara, R., Côrtes, M. C., Fadini, R., Von Matter, S., Leite, A. B., ... Jordano, P, (2013). Functional extinction of birds drives rapid evolutionary changes in seed size. Science, 340, 1086-1090. https:// doi.org/10.1126/science.1233774

García-Callejas, D., Molowny-Horas, R., \& Araújo, M. B. (2018). Multiple interactions networks: Towards more realistic descriptions of the web of life. Oikos, 127, 5-22.

Georgelin, E., \& Loeuille, N. (2016). Evolutionary response of plant interaction traits to nutrient enrichment modifies the assembly and structure of antagonistic-mutualistic communities. Journal of Ecology, 104, 193-205. https://doi.org/10.1111/1365-2745.12485

Godoy, O., Bartomeus, I., Rohr, R. P., \& Saavedra, S. (2018). Towards the integration of niche and network theories. Trends in Ecology \& Evolution, 33(4), 287-300. https://doi.org/10.1016/j.tree.2018.01.007

Gracia-Lázaro, C., Hernández, L., Borge-Holthoefer, J., \& Moreno, Y. (2018). The joint influence of competition and mutualism on the biodiversity of mutualistic ecosystems. Scientific Reports, 8, 9253. https://doi.org/10.1038/s41598-018-27498-8

Gravel, D., Massol, F., \& Leibold, M. A. (2016). Stability and complexity in model meta-ecosystems. Nature Communications, 7, 12457. https:// doi.org/10.1038/ncomms12457

Green, J. L., Hastings, A., Arzberger, P., Ayala, F. J., Cottingham, K. L., Cuddington, K., ... Neubert, M. (2005). Complexity in ecology and conservation: Mathematical, statistical, and computational challenges. AIBS Bulletin, 55, 501-510.

Guimarães, P. R. Jr, Pires, M. M., Jordano, P., Bascompte, J., \& Thompson, J. N. (2017). Indirect effects drive coevolution in mutualistic networks. Nature, 550, 511. https://doi.org/10.1038/nature24273

Hautier, Y., Niklaus, P. A., \& Hector, A. (2009). Competition for light causes plant biodiversity loss after eutrophication. Science, 324, 636-638. https://doi.org/10.1126/science.1169640

Hempson, G. P., Archibald, S., \& Bond, W. J. (2015). A continent-wide assessment of the form and intensity of large mammal herbivory in africa. Science, 350, 1056-1061. https://doi.org/10.1126/science.aac7978

Hiiesalu, I., OePIK, M., Metsis, M., Lilje, L., Davison, J., Vasar, M., ... Paertel, M. (2012). Plant species richness belowground: Higher richness and new patterns revealed by next-generation sequencing. Molecular Ecology, 21, 2004-2016. https://doi. org/10.1111/j.1365-294X.2011.05390.x

Holling, C. S. (1959). Some characteristics of simple types of predation and parasitism. The Canadian Entomologist, 91, 385-398. https://doi. org/10.4039/Ent91385-7

Huang, X., Gao, J., Buldyrev, S. V., Havlin, S., \& Stanley, H. E. (2011). Robustness of interdependent networks under targeted attack. Physical Review E, 83, 065101. https://doi.org/10.1103/ PhysRevE.83.065101

Hutchinson, M. C., Cagua, E. F., \& Stouffer, D. B. (2017). Cophylogenetic signal is detectable in pollination interactions across ecological scales. Ecology, 98, 2640-2652. https://doi.org/10.1002/ecy.1955

Jordano, P. (2016). Sampling networks of ecological interactions. Functional Ecology, 30, 1883-1893.

Kartzinel, T. R., Chen, P. A., Coverdale, T. C., Erickson, D. L., KressWJ, K. M. L., Rubenstein, D. I., ... Pringle, R. M. (2015). Dna 
metabarcoding illuminates dietary niche partitioning by african large herbivores. Proceedings of the National Academy of Sciences of the United States of America, 112, 8019-8024. https://doi.org/10.1073/ pnas.1503283112

Kéfi, S., Miele, V., Wieters, E. A., Navarrete, S. A., \& Berlow, E. L. (2016). How structured is the entangled bank? the surprisingly simple organization of multiplex ecological networks leads to increased persistence and resilience. PLoS Biology, 14, e1002527. https://doi. org/10.1371/journal.pbio.1002527

Kempel, A., Schädler, M., Chrobock, T., Fischer, M., \& van Kleunen, M. (2011). Tradeoffs associated with constitutive and induced plant resistance against herbivory. Proceedings of the National Academy of Sciences of the United States of America, 108, 5685-5689. https://doi. org/10.1073/pnas.1016508108

Kivelä, M., Arenas, A., Barthelemy, M., Gleeson, J. P., Moreno, Y., \& Porter, M. A. (2014). Multilayer networks. Journal of Complex Networks, 2, 203-271.

Laigle, I., Aubin, I., Digel, C., Brose, U., Boulangeat, I., \& Gravel, D. (2018). Species traits as drivers of food web structure. Oikos, 127, 316-326. https://doi.org/10.1111/oik.04712

Lanuza, J. B., Bartomeus, I., \& Godoy, O. (2018). Opposing effects of floral visitors and soil conditions on the determinants of competitive outcomes maintain species diversity in heterogeneous landscapes. Ecology Letters, 21, 865-874.

Laverty, T. M., \& Plowright, R. (1985). Competition between hummingbirds and bumble bees for nectar in flowers of impatiens biflora. Oecologia, 66, 25-32. https://doi.org/10.1007/BF00378548

Lawton, J. H. (1999). Are there general laws in ecology? Oikos, 177-192. https://doi.org/10.2307/3546712

Lindstedt, S. L., Miller, B. J., \& Buskirk, S. W. (1986). Home range, time, and body size in mammals. Ecology, 67, 413-418.

Liu, R. R., Eisenberg, D. A., Seager, T. P., \& Lai, Y. C. (2018). The weak interdependence of infrastructure systems produces mixed percolation transitions in multilayer networks. Scientific Reports, 8, 2111. https://doi.org/10.1038/s41598-018-20019-7

Losapio, G., Pugnaire, F. I., O'Brien, M. J., \& Schöb, C. (2018). Plant life history stage and nurse age change the development of ecological networks in an arid ecosystem. Oikos. https://doi.org/10.1111/ oik.05199

MacArthur, R., \& Levins, R. (1967). The limiting similarity, convergence, and divergence of coexisting species. The American Naturalist, 101, 377-385.

Maron, J. L., \& Vilà, M. (2001). When doherbivores affect plantinvasion? evidence for the natural enemies and biotic resistance hypotheses. Oikos, 95, 361-373. https://doi.org/10.1034/j.1600-0706.2001.950301.x

May, R. M. (1972). Will a large complex system be stable? Nature, 238, 413. https://doi.org/10.1038/238413a0

McDonald, R. A. (2002). Resource partitioning among british and irish mustelids. Journal of Animal Ecology, 71, 185-200. https://doi. org/10.1046/j.1365-2656.2002.00588.x

McGill, B. J., Enquist, B. J., Weiher, E., \& Westoby, M. (2006). Rebuilding community ecology from functional traits. Trends in Ecology \& Evolution, 21, 178-185. https://doi.org/10.1016/j.tree.2006.02.002

Millennium Ecosystem Assessment (2005). Ecosystems and human wellbeing. Washington, DC: Island Press.

Moens, M. A., Valkiūnas, G., Paca, A., Bonaccorso, E., Aguirre, N., \& Pérez-Tris, J. (2016). Parasite specialization in a unique habitat: hummingbirds as reservoirs of generalist blood parasites of Andean birds. Journal of Animal Ecology, 85, 1234-1245. https://doi. org/10.1111/1365-2656.12550

Norouzzadeh, M. S., Nguyen, A., Kosmala, M., Swanson, A., Palmer, M. S., Packer, C., \& ... 2018). Automatically identifying, counting, and describing wild animals in camera-trap images. Proceedings of the National Academy of Sciences of the United States of America, 15(25), E5716-E5725. https://doi.org/10.1073/pnas.1719367115
Novak, M., Yeakel, J. D., Noble, A. E., Doak, D. F., Emmerson, M., Estes, J. A., ... Wootton, J. T. (2016). Characterizing species interactions to understand press perturbations: What is the community matrix? Annual Review of Ecology, Evolution, and Systematics, 47, 409-432.

Olesen, J. M., Bascompte, J., Dupont, Y. L., Elberling, H., Rasmussen, C., \& Jordano, P. (2011). Missing and forbidden links in mutualistic networks. Proceedings of the Royal Society B: Biological Sciences, 278, 725-732. https://doi.org/10.1098/rspb.2010.1371

Olesen, J. M., Bascompte, J., Dupont, Y. L., \& Jordano, P. (2007). The modularity of pollination networks. Proceedings of the National Academy of Sciences of the United States of America, 104, 19891-19896. https:// doi.org/10.1073/pnas.0706375104

Paine, R. T. (1988). Road maps of interactions or grist for theoretical development? Ecology, 69, 1648-1654. https://doi.org/10.2307/ 1941141

Pascual, M., Dunne, J. A. (2006). Ecological networks: Linking structure to dynamics in food webs. Oxford, UK: Oxford University Press.

Pilosof, S., He, Q., Tiedje, K. E., Ruybal-Pesantez, S., Day, K., \& Pascual, M. (2018). Competition for hosts modulates vast antigenic diversity to generate persistent strain structure in plasmodium falciparum. Biorxiv, 406546.

Pilosof, S., Porter, M. A., Pascual, M., \& Kéfi, S. (2017). The multilayer nature of ecological networks. Nature Ecology \& Evolution, 1, 0101. https://doi.org/10.1038/s41559-017-0101

Poelen, J. H., Simons, J. D., \& Mungall, C. J. (2014). Global biotic interactions: An open infrastructure to share and analyze species-interaction datasets. Ecological Informatics, 24, 148-159. https://doi. org/10.1016/j.ecoinf.2014.08.005

Poisot, T., Canard, E., Mouillot, D., Mouquet, N., \& Gravel, D. (2012). The dissimilarity of species interaction networks. Ecology Letters, 15, 1353-1361. https://doi.org/10.1111/ele.12002

Poisot, T., Baiser, B., Dunne, J. A., Kéfi, S., Massol, F., Mouquet, N., ... Gravel, D. (2016). mangal-making ecological network analysis simple. Ecography, 39, 384-390. https://doi.org/10.1111/ecog.00976

Ponisio, L. C., Gaiarsa, M. P., \& Kremen, C. (2017). Opportunistic attachment assembles plant-pollinator networks. Ecology Letters, 20, 12611272. https://doi.org/10.1111/ele.12821

Ramankutty, N., Evan, A. T., Monfreda, C., \& Foley, J. A. (2008). Farming the planet: 1. Geographic distribution of global agricultural lands in the year 2000. Global Biogeochemical Cycles, 22, n/a-n/a. https://doi. org/10.1029/2007GB002952

Reis, S. D., Hu, Y., Babino, A., Andrade, J. S. Jr, Canals, S., Sigman, M., \& Makse, H. A. (2014). Avoiding catastrophic failure in correlated networks of networks. Nature Physics, 10, 762. https://doi.org/10.1038/ nphys3081

Rosenheim, J. A. (2001). Source-sink dynamics for a generalist insect predator in habitats with strong higher-order predation. Ecological Monographs, 71, 93-116. https://doi.org/10.2307/3100046

Rudolf, V., \& Lafferty, K. D. (2011). Stage structure alters how complexity affects stability of ecological networks. Ecology Letters, 14, 75-79. https://doi.org/10.1111/j.1461-0248.2010.01558.x

Sale, P. F. (2013). The ecology of fishes on coral reefs. San Diego, CA: Elsevier.

Salo, P., Korpimäki, E., Banks, P. B., Nordström, M., \& Dickman, C. R. (2007). Alien predators are more dangerous than native predators to prey populations. Proceedings of the Royal Society B: Biological Sciences, 274, 1237-1243. https://doi.org/10.1098/ rspb.2006.0444

Sauve, A., Thébault, E., Pocock, M. J., \& Fontaine, C. (2016). How plants connect pollination and herbivory networks and their contribution to community stability. Ecology, 97, 908-917.

Singh, J., \& Yadava, P. (1974). Seasonal variation in composition, plant biomass, and net primary productivity of a tropical grassland at kurukshetra, india. Ecological Monographs, 44, 351-376. https://doi. org/10.2307/2937034 
Stiles, F. G. (1978). Possible specialization for hummingbird-hunting in the tiny hawk. The Auk, 95, 550-553.

Thébault, E., \& Fontaine, C. (2010). Stability of ecological communities and the architecture of mutualistic and trophic networks. Science, 329, 853-856.

Thompson, J. N. (2005). The geographic mosaic of coevolution. Chicago, IL: University of Chicago Press.

Timóteo, S., Correia, M., Rodríguez-Echeverría, S., Freitas, H., \& Heleno, R. (2018). Multilayer networks reveal the spatial structure of seed-dispersal interactions across the great rift landscapes. Nature Communications, 9, 140. https://doi.org/10.1038/s41467-017-02658-y

Toju, H., \& Baba, Y. G. (2018). Dna metabarcoding of spiders, insects, and springtails for exploring potential linkage between above-and belowground food webs. Zoological Letters, 4, 4.
Vázquez, D. P., Morris, W. F., \& Jordano, P. (2005). Interaction frequency as a surrogate for the total effect of animal mutualists on plants. Ecology Letters, 8, 1088-1094. https://doi. org/10.1111/j.1461-0248.2005.00810.x

How to cite this article: Hutchinson MC, Bramon Mora B, Pilosof S, et al. Seeing the forest for the trees: Putting multilayer networks to work for community ecology. Funct Ecol. 2019;33:206-217. https://doi.org/10.1111/1365$\underline{2435.13237}$ 